\title{
TRIBUNAD DE ACTUALIDAD
}

\section{LAS NUEVAS TECNOLOGÍAS Y LA ADMINISTRACIÓN DE JUSTICIA*. Un nuevo paradigma para los juristas, en especial los jueces}

NEW TECHNOLOGIES AND THE ADMINISTRATION OF JUSTICE. A new paradigm for jurists, especially judges

Ángela García Jiménez

Investigadora

Universidad de Málaga

angelagarciajimenez10@uma.es ORCID 0000-0001-8668-9280

Recepción de trabajo: 02-04-2021 - Aceptación: 03-04-2021

-1. INTRODUCCIÓN. $\approx$ 2. TECNOLOGÍAS DE LA INDUSTRIA 4.0. *3. EL "EFECTO SUSTITUCIÓN" ENTRE LOS JURISTAS. ESPECIAL REFERENCIA A LA SUSTITUCIÓN DE LOS JUECES. 4. CONCLUSIONES. 


\section{RESUMEN}

La Industria 4.0 ha introducido un cambio trascendental en todos los ámbitos de nuestra vida, a unas velocidades cada vez más extremas. En especial, el mundo laboral ha sido objeto de una profunda transformación, con la posible sustitución de los trabajadores y la implementación de las nuevas tecnologías. El objetivo es analizar cómo se reflejan estos efectos en relación con los trabajos del mundo jurídico y en qué medida vienen a afectar a los juristas. En concreto, centraremos el estudio en la posibilidad de sustituir a los jueces y magistrados por máquinas, para comprobar si es viable encontrarnos en un futuro con una total deshumanización de la Administración de Justicia.

PALABRAS CLAVE: Industria 4.0, sustitución, juristas, robotización, jueces, algoritmos.

\section{ABSTRACT}

Industry 4.0 has introduced a momentous change in all areas of our lives. In particular, the world of work has undergone a profound transformation, with the posible replacement of workers and the implementation of new technologies. The purpose is to analyze how these effects are reflected in the legal work and how it affects jurists. Specifically, we will focus on the possibility of replacing judges and magistrates with machines, to determine if it is feasible to find ourselves in the future with a total deshumanization of de Administration of Justice.

KEYWORDS: Industry 4.0, replacement, jurists, robotization, judges, algorithms. 


\section{INTRODUCCIÓN}

El nuevo paradigma tecnológico en el que nos encontramos inmersos desde hace años ha supuesto un cambio radical en la forma en que vivimos, afectando a todas las esferas de la vida humana. La materialización de esta transformación digital y tecnológica se produce en el fenómeno conocido como la Cuarta Revolución Industrial, cuya terminología ha ido afianzándose a lo largo de los años, originalmente introducida por el Fundador y Presidente Ejecutivo del Foro Económico Mundial _FEM en adelante_, Klaus Schwab. Especialmente esclarecedora es en este sentido la definición otorgada por el Ministerio de Industria, Energía y Turismo, entendiendo por Cuarta Revolución Industrial aquella "que se basa en la disponibilidad en tiempo real de toda la información relevante al producto, proporcionada por una red accesible en toda la cadena de valor. Esto se logra a través de la digitalización y la unión de todas las unidades productivas de una economía. Para ello, es necesaria la fusión de tecnologías tales como Internet de las cosas (IOT2), computación y cloud, big data y ciberseguridad, así como las complementarias: móvil, analytics, M2M, impresión 3D, robótica y comunidad/compartición"s.

A partir de dicho concepto, han surgido otros que pretenden englobar el mismo fenómeno, como es el de Industria 4.04. Este nuevo modelo de producción industrial destaca, entre otros aspectos, por el uso de las KETs ${ }^{5}$, traducidas como "tecnologías facilitadoras", proporcionando elementos tecnológicos que permiten el desarrollo de nuevos productos, procesos y servicios. Las nuevas tecnologías empleadas en esta Industria 4.0 son muy variadas, lo que nos hace destacar especialmente como tecnologías facilitadoras las siguiente: Internet de las Cosas, análisis de datos y big data, inteligencia artificial, computación cognitiva, sistemas ciberfísicos, robotización, realidad virtual, drones, machine learning, blockchain ${ }^{6}$ o ciberseguridad, entre otros ${ }^{7}$.

1 Destaca así su obra La cuarta revolución industrial. Debate. 2016

2 Abreviatura de Internet of Things en inglés. IdC en español.

3 Ministerio de Industria, Energía y Turismo, "Industria conectada 4.0: La transformación digital de la industria española", Ministerio de Industria, Energía y Turismo, Madrid, 2015, pág. 114.

4 Martínez Aguiló, J. Industria 4.0: La transformación digital en la Industria. Editorial UOC. 2019. Obtenido de https://elibro--net.uma.debiblio.com/es/Ic/uma/ titulos/113336

$5 \quad$ Key Enabling Technologies, KETs, por sus siglas en inglés.

6 El FEM sen̂ala que el blockchain permite conseguir una mayor confianza y transparencia a través de la descentralización, criptografía y la creación de nuevos incentivos. Es más conocido como soporte digital de Bitcoin, convirtiéndose en una tecnología esencial en muchas áreas, entre las que destaca el sector financiero en el que se utiliza como medio para reemplazar sistemas de pago costosos e ineficientes, pudiendo a su vez remodelar cadenas de suministro mediante la combinación de Internet de las Cosas y la Inteligencia Artificial. Recuperado de: https://intelligence.weforum.org/topics/alGb00000038qmPEA Q? tab=publications

7 Para MARTíN ROBLES, J., estas tecnologías son: biotecnología, nanotecnología, Inteligencia Artificial, robótica, impresión 3D, nuevos materiales, realidad virtual y big data.

Martín Robles, J., Futurizable, Ediciones Puertollano, 2017. 


\section{TECNOLOGIAS DE LA INDUSTRIA 4.0}

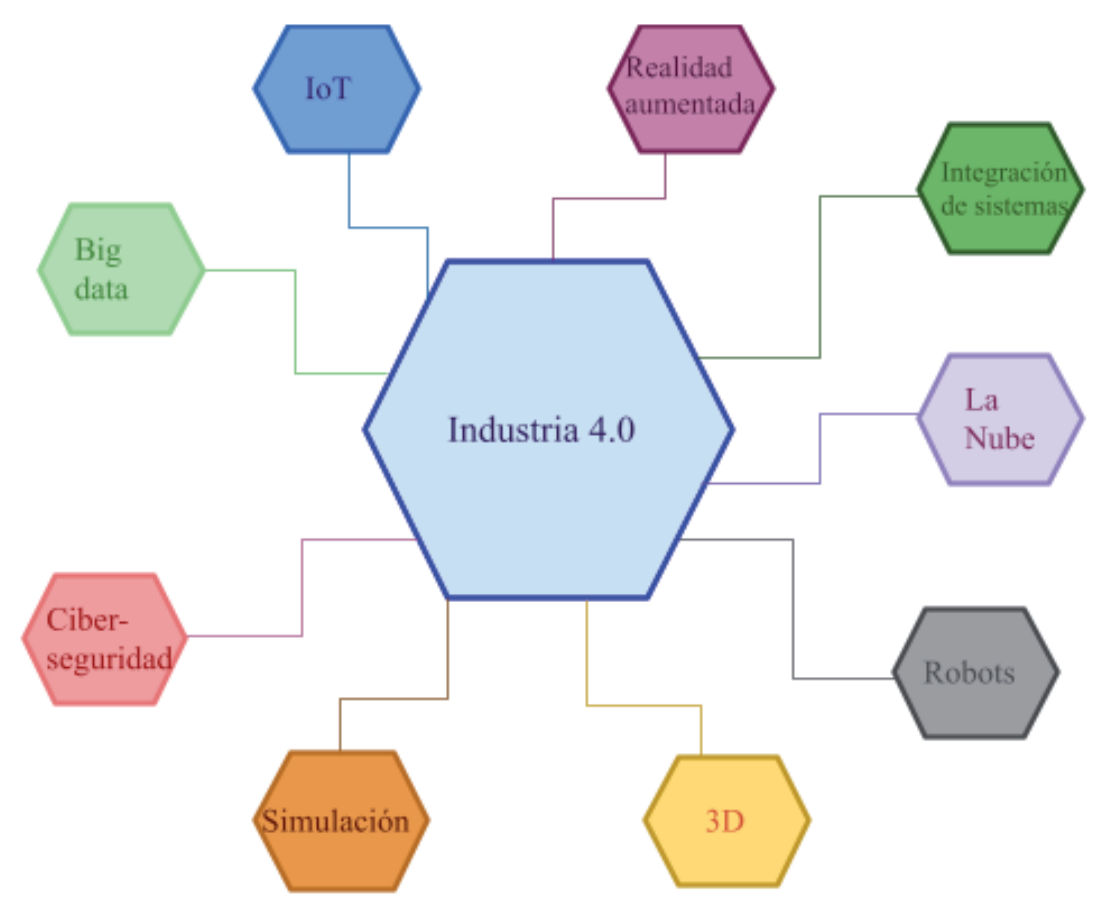

Fig.l. Fuente: Martín Robles, J. Futurizable. Ediciones Puertollano, 2017. Véase también: Masala, A. "What Will the Fourth Industrial Revolution Require?", IREF Europa, disponible en: https://en.irefeurope.org/Publications/Online-Articles/article/What-Will-The-Fourth-Industrial-Revolution-Require

Ciertos autores ${ }^{8}$ han destacado especialmente dos figuras fundamentales que configuran esta revolución tecnológica: la digitalización y la robotización, de manera que se caracteriza por la tecnología suplementaria al trabajo humano de forma automatizada, como así ha sido establecido por el $\mathrm{FEM}^{9}$ en su insistencia sobre la profunda transformación social que ha supuesto la implantación de esta tecnología.

De esta manera, la Cuarta Revolución Industrial se ha convertido en un elemento clave de todos los ámbitos del ser humano, sin que podamos excluir ni mucho menos el ámbito profesional y laboral. Sin perjuicio de los efectos intrínsecos que produce esta nueva tecnología en el desarrollo de las

Entre ellos, Gómez Salado, M.Á., La cuarta revolución industrial y su impacto sobre la productividad, el empleo y las relaciones juridico-laborales: Desafíos tecnológicos del siglo XXI, Thomson Reuters Aranzadi, Cizur Menor, 2021, pág. 44 y ss. Véase también Lahera Sánchez, A., "Digitalización, robotización, trabajo y vida: cartografías, debates y prácticas", Cuaderno de Relaciones Laborales, vol. 37, n², 2019, pp.249-273.

9 Foro Económico Mundial, "Fourth Industrial Revolution", disponible en: https://intelligence.weforum.org/topics/alGb0000001RIhBEAW?tab=publications 
LAS NUEVAS TECNOLOGÍAS Y LA ADMINISTRACIÓN DE JUSTICIA

personas y en el quehacer cotidiano, debemos centrar nuestros esfuerzos en analizar el tremendo impacto que la Industria 4.0 produce en los empleos, las empresas y la forma en la que hasta ahora venía desarrollándose el trabajo no solo a nivel nacional, sino a escala global incentivado por la globalización y las economías plurinacionales e interconectadas.

Las nuevas tecnologías suponen un complemento al trabajo humano en muchas ocasiones, lo que implica no poner en riesgo ningún puesto de trabajo a pesar de su uso, pero también es cierto que gracias a los avances actuales, muchas máquinas son capaces de realizar tareas para las que no solo se necesitaba un único trabajador, sino varios, dando lugar a un proceso de robotización y sustitución de la mano de obra que permite conseguir el mismo resultado en menor tiempo. Así, el proceso de robotización ha comenzado ya para muchos empleos, si bien se espera que en las próximas décadas alcance su máxima expresión. Como consecuencia, existe una creciente preocupación por el conocido como "efecto sustitución", es decir, la desaparición de numerosos puestos de trabajo de forma masiva como consecuencia del uso de las nuevas tecnologías, especialmente la robótica.

Este efecto sustitución viene especialmente conectado con aquellos empleos que realizan tareas rutinarias y repetitivas - lo que facilita en mayor medida la automatización de las mismas y su realización por máquinas capaces de ser programas para realizar esos movimientos de manera continuada y sin la necesidad de descansar - de manera que los trabajadores con una cualificación más baja sufren mayores riesgos de ser sustituidos por robots, como lo indican estudios elaborados por el Consejo Económico y Social —CES por sus siglas- que insisten en una recualificación y formación adicional en su Informe 3/2018, "El futuro del Trabajo". No obstante, en la actualidad podemos afirmar una afección de aquellos trabajos más cualificados, que pueden venir siendo sustituidos por el uso de algoritmos.

\section{EL "EFECTO SUSTITUCIÓN" ENTRE LOS JURISTAS. ESPECIAL REFERENCIA A LA SUSTITUCIÓN DE LOS JUECES}

Resulta objeto de intenso debate la posibilidad de utilizar los robots incluso en ciertos ámbitos en los que consideramos el trabajo humano imprescindible y esencial, principalmente en aquellos en los que no existe una solución a la que puedan llegar estas máquinas a través de los datos registrados, lo que supone que, a pesar de tratarse de una tecnología cada vez más avanzada y compleja, la toma de decisiones a la que es capaz no abarcaría ciertos aspectos.

Este planteamiento se ha plasmado en relación al ámbito de la Justicia, afectando cada vez más a los juristas que se ven sustituidos por sistemas de previsibilidad y justicia predictiva10, especialmente

10 Oliva Santos, A., "Giustizia predittiva, interpretazione matematica delle norme, sentenze robotiche e la vecchia storia del "Justizklavier"'", Riv. trim. dir. proc. civl, n³, 2019, pp. 883 y ss. 
LAS NUEVAS TECNOLOGÍAS Y LA ADMINISTRACIÓN DE JUSTICIA

los jueces, como así han señalado ciertos autores. Nos encontramos cada día con más titulares que rezan de esta forma: "Los bufetes admiten que los robots ya suplen algunos abogados"1 como señalaba El País hace ya dos años, en un artículo donde se centran en analizar el reemplazo del personal jurídico en las principales firmas inglesas, desde los abogados cualificados a los conocidos como "paralegales", cuya función es asesorar a otros letrados.

El informe sobre Legaltech — que analiza el uso de la tecnología en este ámbito— de "The Law Society" indica que se está quintuplicando el uso de esta nueva tecnología en los bufetes, siendo especialmente relevante la automatización. Son destacables a su vez las ventajas que implica esta modernización de las firmas, en la medida en que los abogados pueden optimizar su trabajo, como indica Javier de Cendra, decano del IE Law School en su declaración para Cinco Días, quien considera que "la automatización de parte de los servicios jurídicos liberalizará entre el 30 y el $60 \%$ del tiempo de los abogados dedicados a tareas de poco valor"12.

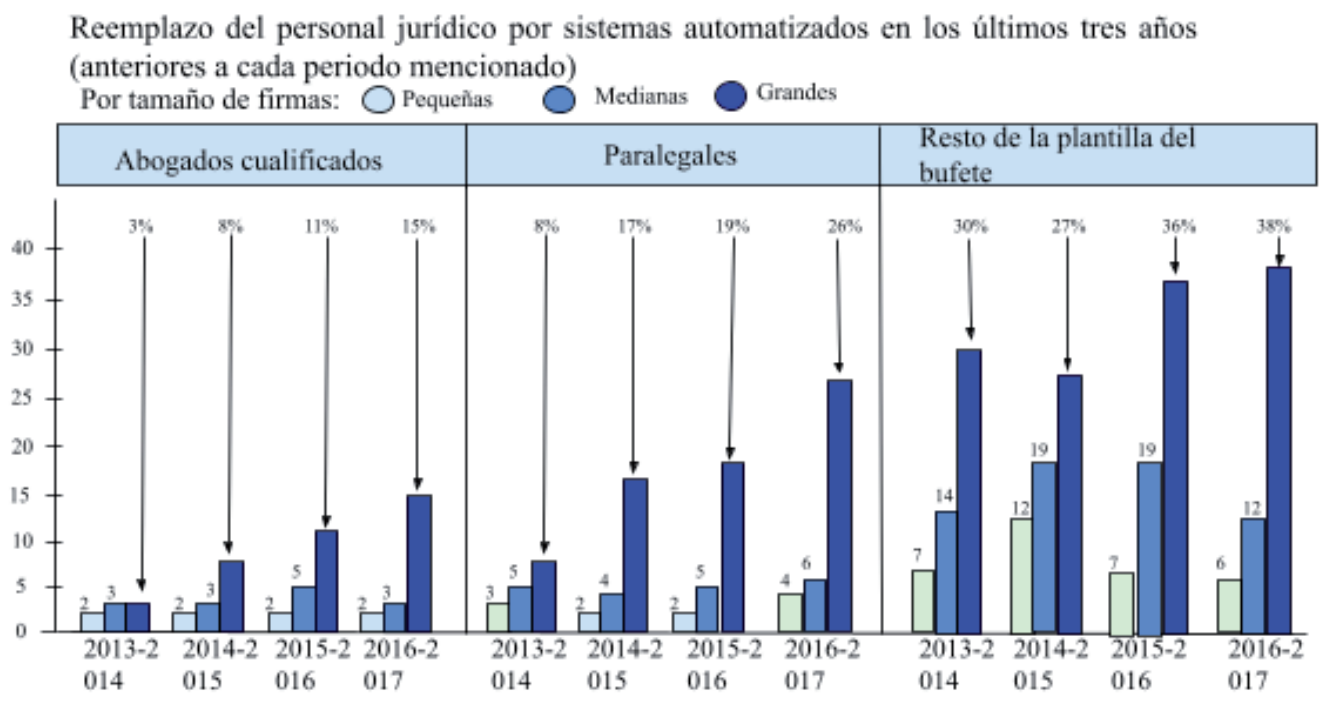

Fuente: Introduction to LawTech. A practical guide to legal technologie 2019. The Law Society

Sin perjuicio de la profunda transformación transversal que se produce en el mundo jurídico, en este trabajo nos centraremos especialmente en el fuerte impacto que esta nueva era tecnológica está suponiendo para la realización de la actividad jurídica propia de los jueces y magistrados, con la implantación de sistemas de Inteligencia Artificial en los juzgados en sustitución de aquellos pro-

En España, en materia de justicia preventiva contamos con "Jurimetría", una plataforma elaborada por Wolters Kluver. A través de la misma, es posible poder consultar desde las posibilidades de éxito en un caso hasta cómo se debe argumentar cierta pretensión.

11 Del Águila Barbero, P., "Los bufetes admiten que los robots ya suplen algunos abogados", Cinco Días, El País, 8 de diciembre de 2019. Recuperado de: https:// cincodias.elpais.com/cincodias/2019/12/03/legal/1575388049_051347.html

12 Ibídem 
LAS NUEVAS TECNOLOGÍAS Y LA ADMINISTRACIÓN DE JUSTICIA

fesionales del Derecho sobre los que hasta el momento depositábamos la confianza de una buena Administración de Justicia.

De esta forma, ello puede conllevar la posibilidad de resolver un juicio mediante algoritmos ${ }^{13}$. La resolución de pequeñas reclamaciones por robots mediante el uso de la Inteligencia Artificial —en adelante IA - es ya una realidad palpable en algunos países del mundo, como Estonia, China o Estados Unidos ${ }^{14}$. A través de la IA, una máquina, normalmente un ordenador es capaz de realizar tareas para las que un ser humano necesitaría un proceso de razonamiento, mediante el tratamiento de datos introducidos en el sistema.

Como hemos mencionado, es obvio que la decisión a la que pueda llegar una máquina en casos como el que aquí analizamos puede dar lugar a la comisión de errores, lo que obviamente también ocurre en el caso de las decisiones humanas. No obstante, los errores en que puede incurrir uno y otro no son contemplados de la misma forma, planteándose así la injusticia de las decisiones que pueda tomar una máquina, por la falta de ese elemento humano al que nos referíamos, donde esa subjetivación resulta clave en muchas ocasiones, puesto que no todos los procesos son iguales, ni todas las circunstancias a tener en cuenta, por lo que carecen de discernimiento.

De esta manera, el "machine learning" como capacidad de una máquina de aprender y mejorar su capacidad mediante su experiencia, nunca podría equipararse a la capacidad de un experto jurídico para poder enjuiciar y valorar todos los extremos de un caso. A su vez, muchos han puesto en evidencia un riesgo esencial del funcionamiento de estos algoritmos, como es el tipo de datos que se introducen por el programador en estos sistemas. El tratamiento de ciertos datos puede poner de manifiesto los propios sesgos discriminatorios cuya tendencia se ha seguido por aquellos responsables de hacer funcionar estas máquinas. En consecuencia, los resultados a los que se puede llegar a través de estos algoritmos pueden llegar a encauzarse hacia posturas injustas, partiendo del carácter de la información que poseen para solucionar los casos que se plantean.

No obstante, ello no supone no poder afirmar las ventajas que supondría esta automatización dentro de los procesos, principalmente para garantizar la uniformidad de las decisiones, la agilización de una justicia excesivamente lenta como la que vivimos y la facilidad para controlar las decisiones.

Los motivos que llevan a considerar una ventaja esta administración de justicia son: por un lado, la eficiencia a la que nos referíamos en el párrafo anterior, con la descongestión de nuestro sistema de justicia; por otro lado, permite garantizar mayor seguridad jurídica. La cuestión está en determinar si ese algoritmo puede abarcar la posibilidad de interpretar las normas, de manera que la calidad de la tarea robótica fuese la misma que la humana, respetando en todo caso la tutela judicial efec-

13 Entendiéndose el mismo como "la capacidad de una máquina -propiamente un ordenador o un robot- de correlacionar grandes cantidades de información y de datos, según una fórmula determinada para finalmente dar el resultado previsto y requerido".

Battelli, E., "La decisión robótica: algoritmos, interpretación y justicia predictiva", Revista de Derecho Privado, n 40, enero-junio 2020, pp. 45-86.

Salom Lucas, A., "Su sentencia, gracias", El Derecho, 2020, disponible en: https://elderecho.com/su-sentencia-gracias 
LAS NUEVAS TECNOLOGÍAS Y LA ADMINISTRACIÓN DE JUSTICIA

tiva. Como indicamos, la ley no siempre debe y puede seguirse en su tenor literal, en la medida en que no existe una única solución aceptable, por lo que la interpretación constituye uno de los límites fundamentales a los que hasta el momento la tecnología robótica no ha sido capaz de enfrentarse, lo que retrasa en cierta medida la posible sustitución masiva de los jueces.

Respecto a la posible sustitución de los jueces, muchos han rechazado esta posibilidad, defendiendo la idea de la complementariedad a la que nos referimos con anterioridad, lo que implica que debe usarse como instrumento, pero sin eliminar la figura del magistrado. Esto se demuestra en la creciente digitalización de la justicia que hemos vivido en los últimos años ${ }^{15}$, aunque hasta el momento la robótica no ha tenido un papel preponderante en la administración de justicia.

Por ello, el uso de estos sistemas constituye más que una amenaza una ayuda, especialmente relevante a la hora de consultar bases de datos, filtrar la información y clasificarla, de manera que el juez pueda emplear esta tecnología como herramienta en el ejercicio de sus funciones. De esta forma, "el sistema experto es a la vez un bastón de seguridad para el caminante intelectual del Derecho y una exigencia a caminar cada vez más y más atrevidamente, un apoyo del razonamiento del juez y un desafío a su capacidad intelectual"16.

El beneficio innegable de esta justicia robótica lo encontramos en la obtención de una respuesta exacta y objetiva ${ }^{17}$, de manera que la solución a cada caso sería homogénea en la medida en que los hechos fueran los mismos, lo que permite asegurar una solución al asunto antes incluso de que el mismo finalice. No obstante, la decisión adoptada por un robot solo puede adoptarse atendiendo a los datos introducidos en el mismo, mientras que el juez tiene en cuenta todas aquellos elementos fácticos que se le hayan representado, dependiendo la solución en ambos casos de la exactitud de los hechos que los terceros proporcionen a uno o a otro ${ }^{18}$.

\section{CONCLUSIONES}

En definitiva, la solución de un juez humano no está exenta de errores, pero tampoco lo está aquella a la que podría llegar un juez robot, por lo que la sustitución no se concibe, al menos a corto plazo,

Asi lo demuestra el informe de la Comisión Europea para la Eficiencia de la Justicia (CEPE)), siendo uno de los más avanzados de Europa, con instrumentos de telecomunicación como LexNet.

Ministerio de Justicia, "España a la vanguardia en el proceso de digitalización de la Justicia Europea", LexNet Justicia, 2020. Disponible en: https://lexnetjusticia.gob.es/blog/-/blogs/espana-a-la-vanguardia-en-el-proceso-de-digitalizacion-de-la-justicia-en-europa

16 Trazegnies, de F., "'Seguirán existiendo jueces en el futuro? El razonamiento judicial y la inteligencia artificial. Revista lus et Veritas, n 47, diciembre 2013, página 128.

17 Véase Luciani, M., "La decisione giudiziaria robotica", Riv. Associaz. Italiana Constituzionalisti, nº 3, 2018. Pág. 880.

18 Ibídem 
LAS NUEVAS TECNOLOGÍAS Y LA ADMINISTRACIÓN DE JUSTICIA

como una solución admisible, en especial por la carencia de cualidades humanas irrenunciables en la interpretación y aplicación de las normas. De esta forma, si bien es cierto que no podemos negar la utilidad que los sistemas de IA pueden suponer para la función jurisdiccional, resulta todavía inaceptable la absoluta sustitución del juzgador, cuya capacidad humana y conocimientos jurídicos constituyen un elemento imprescindible en la tarea de juzgar y hacer ejecutar lo juzgado. 